\title{
Tratamiento de la retinopatía del prematuro con bevacizumab intravítreo
}

\author{
ALDO BANCALARI M. ${ }^{1}$, RICARDO SCHADE Y. ${ }^{2}$, RUBÉN PEÑA Z. ${ }^{3}$, NICOLÁS PAVEZ P. ${ }^{3}$ \\ 1. Unidad de Neonatología, Servicio de Pediatría, Hospital Guillermo Grant Benavente. Facultad de Medicina, \\ Universidad de Concepción. \\ 2. Servicio de Oftalmología, Hospital Guillermo Grant Benavente. \\ 3. Interno de Medicina, Facultad de Medicina, Universidad de Concepción.
}

\begin{abstract}
Intravitreal bevacizumab as treatment for retinopathy of prematurity

Introduction: Standard treatment of threshold Retinopathy of Prematurity (ROP) is laser photocoagulation (LPC). Recently, bevacizumab, a vascular endothelial growth factor (VEGF) inhibitor has been used with positive results. Objective: To communicate the response to treatment with bevacizumab in premature newborns with threshold retinopathy. Patients and Method: Very low birth weight (VLBW) infants that received bevacizumab, in monotherapy or associated with LPC, as treatment for threshold ROP, were analyzed. Response to treatment, reinterventions and complications were evaluated. Results: 17 VLBW infants were analyzed with mean gestational age $26.4 \pm 1.7$ weeks and mean birth weight $850 \pm 141 \mathrm{~g}$. All 6 patients treated with bevacizumab in association with LPC did not require reintervention. Of the 11 neonates that only received bevacizumab, 36.3\% needed further treatment. Conclusions: Bevacizumab could be an effective therapy for treatment of threshold ROP. In our experience it presented advantages compared with LPC, since therapy can be carried out without transferring the patient and with no use of general anesthetics. No short term complications were observed with the use of bevacizumab in treated patients.

(Key words: Retinopathy of prematurity; intravitreal injections; bevacizumab, vascular endothelial growth factor).

Rev Chil Pediatr 2013; 84 (3): 300-307
\end{abstract}

\section{RESUMEN}

Introducción: El tratamiento estándar de la Retinopatía del Prematuro (RDP) en etapa umbral es la fotocoagulación con láser (FCL). Recientemente se ha utilizado el bevacizumab, un inhibidor del factor de crecimiento del endotelio vascular (VEGF) con resultados alentadores. Objetivo: Comunicar el resultado del tratamiento con bevacizumab en Recién Nacidos (RN) prematuros con retinopatía en etapa umbral. Pacientes y Método: Se analizaron los RN pretérmino de muy bajo peso (RNMBP) que recibieron bevacizumab, en monoterapia o asociado a FCL, como tratamiento de la RDP en etapa umbral. Se evaluó respuesta al tratamiento, reinter-

Recibido el 20 de octubre de 2012, devuelto para corregir el 18 de diciembre de 2012, segunda versión 04 de febrero de 2013 , aceptado para publicación el 25 de febrero de 2013.

Este trabajo cumple con los requisitos sobre consentimiento /asentimiento informado, comité de ética, financiamiento, estudios animales y sobre la ausencia de conflictos de intereses según corresponda.

Correspondencia a:

Aldo Bancalari Molina

E-mail: aldobancalari@gmail.com 
venciones y complicaciones. Resultados: Se analizaron 17 RNMBP con promedio de edad gestacional 26,4 \pm 1,7 sem y peso de nacimiento de $850 \pm 141 \mathrm{~g}$. Los 6 pacientes tratados con bevacizumab en asociación a FCL no requirieron reintervención. De los 11 neonatos que sólo recibieron bevacizumab, el 36,3\% necesitó posteriormente tratamiento con FCL. Conclusiones: El bevacizumab sería una terapia efectiva en el tratamiento de la RDP umbral. En nuestra experiencia presentó ventajas respecto a la FCL, al ser realizado sin necesidad de traslado del RN ni de uso de anestesia general. No observamos complicaciones a corto plazo con el uso de bevacizumab en los pacientes tratados.

(Palabras clave: Retinopatía del prematuro; inyecciones intravítreas; bevacizumab, factor de crecimiento endotelial).

Rev Chil Pediatr 2013; 84 (3): 300-307

\section{Introducción}

La retinopatía del prematuro (RDP) es una enfermedad proliferativa de la vasculatura retinal que puede ocasionar una pérdida visual severa en Recién Nacidos (RN) prematuros ${ }^{1}$. Es la principal causa de ceguera infantil en los países desarrollados y en vías de desarro$1 \mathrm{o}^{2}$. En Chile un estudio efectuado en escuelas de ciegos demostró que la RDP es también la principal causa de ceguera infantil ${ }^{3}$. En un reporte nacional, la incidencia fue de 58,8\% en los niños con peso de nacimiento inferior a $1.000 \mathrm{~g}$, y de $28,9 \%$ en aquellos con peso entre $1.000 \mathrm{~g}$ y $1.249 \mathrm{~g}^{4}$.

La enfermedad se caracteriza por la proliferación de tejido fibrovascular en el límite entre retina vascular y avascular ${ }^{5}$. En la patogénesis se describen dos fases, la primera caracterizada por un ambiente hiperóxico con bajos niveles circulantes de factor de crecimiento endotelial vascular (VEGF), seguida por una segunda fase de proliferación vascular inducida por hipoxia con elevados niveles de $\mathrm{VEGF}^{6}$. Es en esta segunda fase donde el aumento del VEGF juega un rol fundamental en la progresión de esta enfermedad ${ }^{7}$.

El uso de medicamentos inhibidores del VEGF ha mostrado resultados alentadores como tratamiento de la retinopatía en etapa umbral ${ }^{8}$; como monoterapia o bien como coadyuvante a la fotocoagulación con láser (FCL), que es considerada hasta el momento el tratamiento de elección para la $\mathrm{RDP}^{9}$. Dentro de los inhibidores del VEGF, el bevacizumab es un anticuerpo monoclonal recombinante humanizado, cuyo mecanismo de acción se basa en la unión y en la consiguiente neutralización del VEFG, evitando su asociación a receptores endoteliales ${ }^{10}$.

El bevacizumab ha sido utilizado principalmente como agente antineoplásico en diversos tumores, siendo su uso aprobado para el cáncer de colon, pulmonar, renal y cerebra $1^{11}$. Debido a su mecanismo de acción, se ha explorado su uso en oftalmología, especialmente en la retinopatía diabética y en la degeneración macular exudativa ${ }^{12}$. Últimamente se ha extendido su uso a la retinopatía del prematuro principalmente en etapa umbral, ya sea como tratamiento único o como coadyuvante a la terapia estándar con láser ${ }^{7-9}$.

Los primeros reportes de su uso datan del año 2007 en los que se muestra la experiencia en uno y tres pacientes con resultados positi$\operatorname{vos}^{13,14}$. Esto motivó su uso en nuestro centro desde el año 2008, inicialmente en forma combinada con FCL y posteriormente como monoterapia, en pacientes con RDP en etapa umbral en que no fue posible realizar FCL en primera instancia.

\section{Objetivo}

Comunicar el resultado del tratamiento con bevacizumab intravítreo en recién nacidos prematuros con retinopatía en etapa umbral.

\section{Pacientes y Método}

Se analizaron las historias clínicas de los Recién Nacidos pretérmino de muy bajo peso (RNMBP) en los que se administró bevacizumab (Avastin ${ }^{\circledR}$ ) por presentar RDP, durante el período comprendido entre enero de 2008 y febrero de 2012. Se consideraron los antecedentes demográficos de los pacientes y la 
evolución oftalmológica. En todos los neonatos se obtuvo consentimiento informado de los padres previo al tratamiento, explicándose detalladamente la indicación, forma de administración y posibles complicaciones del fármaco. El uso de bevacizumab en nuestro centro se encuentra aprobado por el Comité de Ética del Hospital.

El examen oftalmológico se inició a las 4 semanas (sem) de nacimiento, por oftalmología indirecta, previa dilatación con tropicamide $0,5 \%$, realizándose control semanal.

La clasificación de RDP se efectuó de acuerdo a la clasificación internacional de retinopatía $^{15}$. Los candidatos para tratamiento fueron aquellos pacientes que alcanzaron retinopatía en etapa 3 plus $(3+)$, y en casos en etapa $2+$, cuando existió compromiso en zona I o II posterior.

Los primeros pacientes tratados en nuestra experiencia con anti-VEGF, correspondieron a recién nacidos prematuros en los que se usó bevacizumab en forma coadyuvante a la FCL, con la finalidad de aumentar el éxito terapéutico y evitar reintervenciones. Luego de la experiencia inicial se utilizó bevacizumab en forma primaria en aquellos prematuros en los que no se pudo utilizar FCL en el momento en que estaba indicada por las siguientes situaciones: dilatación incompleta por rubeosis; enfermedad en zona posterior; y contraindicación de anestesia general por inestabilidad del prematuro.

La sedación del RN se realizó con fentanilo. El globo ocular se anestesió en forma tópica con proparacaína $0,5 \%$, previa asepsia con povidona yodada $5 \%$. Se inyectó bevacizumab a través de pars plana a $2 \mathrm{~mm}$ del limbo, a una dosis de 0,625 mg (figura 1). Posterior a la intervención se indicó profilaxis antibiótica con colirio de tobramicina y dexametasona cada $3 \mathrm{~h}$ por tres días. El medicamento fue administrado en la Unidad de Neonatología del Hospital Guillermo Grant Benavente, en todas las ocasiones por el mismo profesional (RS). El láser utilizado fue Iris Medical ${ }^{\circledR}$ con longitud de onda $232 \mathrm{~nm}$ con 200 miliwatts de potencia de 200 milisegundos de duración con intervalos de 400 milisegundos entre cada disparo. Posterior a la intervención se realizaron controles oftalmológicos seriados, al día siguiente y luego cada semana hasta los 3 meses y posteriormente en forma mensual hasta alcanzar la edad corregida de 1 año. El seguimiento general y neurológico se realizó según cronograma nacional de atención del recién nacido.

Con un fin descriptivo, se dividieron a los pacientes en dos grupos: aquellos que recibieron bevacizumab como coadyuvante a la fotocoagulación con láser en el mismo tiempo operatorio (grupo A), y los que recibieron bevacizumab como tratamiento único (grupo B) como monoterapia. En cada paciente se evaluó la respuesta al tratamiento y complicaciones oculares con su uso, así como también la necesidad de reintervención. Se consideraron candidatos a reintervención mediante fotocoagulación con láser, aquellos pacientes que progresaron o se mantuvieron en etapa $2+$ zona posterior o etapa 3 , una vez administrado el bevacizumab.

\section{Resultados}

Se trataron 17 RNMBP, con una edad gestacional y peso de nacimiento promedio $\pm \mathrm{DS}$ de $26,4 \pm 1,7$ sem y $850 \pm 141$ g respectivamente. La edad postnatal al momento de la administración de bevacizumab fue de $8,2 \pm 2,0$ sem. La distribución por género muestra una predominancia del sexo femenino correspondiendo al 64\%. Los datos demográficos indi-

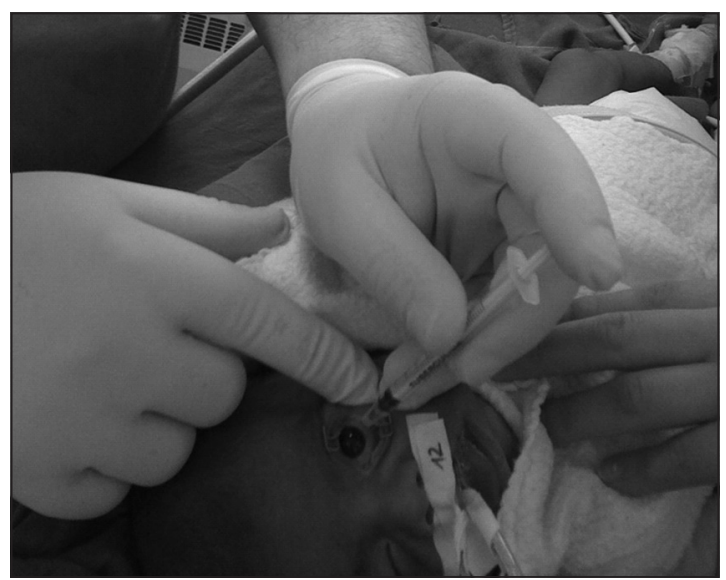

Figura 1. Administración intravítrea de bevacizumab en la Unidad de Neonatología. 
Tabla 1. Características demográficas y compromiso de los pacientes con RDP tratados con bevacizumab

\begin{tabular}{|ccccccclc|}
\hline Paciente & $\begin{array}{c}\text { EG } \\
\text { (sem) }\end{array}$ & Sexo & $\begin{array}{c}\text { Peso } \\
\text { (g) }\end{array}$ & Etapa & Zona & $\begin{array}{c}\text { EG corregida } \\
\text { al tratamiento } \\
\text { (sem) }\end{array}$ & Tratamiento & Reintervención \\
\hline 1 & 26 & M & 840 & $3+$ & 2 & 35 & Bevacizumab + Láser \\
\hline 2 & 26 & F & 850 & $3+$ & 2 & 36 & Bevacizumab \\
\hline 3 & 29 & F & 980 & $3+$ & 3 & 37 & Bevacizumab + Láser \\
\hline 4 & 25 & F & 620 & $3+$ & 1 & 35 & Bevacizumab + Láser \\
\hline 5 & 26 & F & 760 & $3+$ & 3 & 36 & Bevacizumab + Láser \\
\hline 6 & 28 & F & 920 & $3+$ & 3 & 35 & Bevacizumab + Láser \\
\hline 7 & 26 & F & 880 & $3+$ & 2 & 36 & Bevacizumab + Láser & Láser \\
\hline 8 & 27 & F & 900 & $3+$ & 2 & 35 & Bevacizumab \\
\hline 9 & 26 & M & 680 & $3+$ & 3 & 36 & Bevacizumab \\
\hline 10 & 25 & F & 1120 & $2+$ & 2 & 29 & Bevacizumab \\
\hline 11 & 26 & F & 900 & $2+$ & 2 & 34 & Bevacizumab \\
\hline 12 & 25 & M & 720 & $3+$ & 2 & 35 & Bevacizumab \\
\hline 13 & 31 & F & 1020 & $2+$ & 1 & 36 & Bevacizumab \\
\hline 14 & 24 & F & 610 & $3+$ & 2 & 32 & Bevacizumab \\
\hline 15 & 26 & M & 980 & $2+$ & 1 & 35 & Bevacizumab \\
\hline 16 & 27 & M & 890 & $3+$ & 2 & 32 & Bevacizumab \\
\hline 17 & 25 & M & 780 & $3+$ & 2 & 36 & Bevacizumab \\
\hline
\end{tabular}

EG: Edad gestacional; sem: semanas; g: gramos.

vidualizados de cada neonato se presentan en la tabla 1.

El 76,5\% (13/17) de los pacientes se encontraban con RDP en etapa 3+ al momento de administrar el bevacizumab (tabla 1). Los 4 restantes se encontraban en etapa $2+$, uno de ellos presentó hemorragia retinal (paciente 2). La zona más comprometida fue la zona II con un $58,8 \%$ (10/17). En los 17 pacientes analizados, la retinopatía presentó la misma etapa y zona para ambos ojos en 14 casos $(82,4 \%)$ al momento del tratamiento. Los 3 pacientes con compromiso retinal desigual se describen en la tabla 2. El

Tabla 2. Pacientes con compromiso retinal desigual

\begin{tabular}{|cccl|}
\hline Paciente & OD & OI & Conducta inicial \\
\hline 4 & RDP 3 + ZI & RDP 3 + ZII & Bevacizumab + Láser \\
7 & RDP 3 + ZII & RDP 3 + ZIII & Bevacizumab + Láser \\
\hline 13 & RDP 2 + ZI & RDP 2 + ZII & Bevacizumab \\
\hline
\end{tabular}

OD: Ojo derecho; OI: Ojo izquierdo; Z: zona. tratamiento efectuado fue similar en ambos ojos, independiente del compromiso ocular asimétrico.

Ninguno de los pacientes del grupo A $(\mathrm{n}=6)$ requirió reintervención con FCL ni una nueva dosis de bevacizumab. De los 11 neonatos del grupo $\mathrm{B}$, que sólo recibieron bevacizumab, en 4 de ellos $(36,4 \%)$ se necesitó posteriormente efectuar tratamiento con FCL (tabla 1). Las reintervenciones fueron necesarias por progresar la RDP a etapa 3 en su evolución, habiéndose documentado previamente una fase de regresión a etapa 1 . Las reintervenciones fueron realizadas en 3 pacientes en la tercera semana y en uno a las nueve semanas de administrado el fármaco, correspondiendo a pacientes que presentaban compromiso de la zona II. El 63,6\% restante ( 7 pacientes) se consideró tratado con la inyección intravítrea.

La administración más precoz de bevacizumab como tratamiento único fue a las 4 sem de vida, correspondiendo al $\mathrm{RN}$ de ma- 
yor peso $(1.120 \mathrm{~g})$ y la más tardía a las $10 \mathrm{sem}$ siendo un RN de $620 \mathrm{~g}$ al nacer.

Al realizar los controles oftalmológicos seriados, se observó una evolución satisfactoria y no se encontraron complicaciones asociadas como hifema, endoftalmitis, desprendimiento de retina o catarata. No obstante, en el control al año del paciente 14 (tabla 1) se observó tortuosidad vascular con leve tracción macular en ojo derecho. Fallecieron 2 pacientes (1 y 4; tabla 1) a los 3 y 5 meses de edad postnatal; ambos por sepsis nosocomial y falla multiorgánica.

\section{Discusión}

En nuestra serie el tratamiento con bevacizumab se inició el año 2008 realizado junto a la FCL con la finalidad de aumentar el espectro de pacientes tratados con éxito y disminuir la inflamación y consecuente daño retinal provocado por el tratamiento con láser. Posteriormente, con mayor experiencia, asociado a la ausencia de efectos colaterales y nueva evidencia científica ${ }^{12-14,16}$, se inició el tratamiento en etapa umbral con bevacizumab, en casos seleccionados. Cabe hacer notar que, fisiopatológicamente, la retinopatía del prematuro se caracteriza por la proliferación vascular mediada por niveles altos de VEFG, por lo que al utilizar bevacizumab se pretende hacer un tratamiento dirigido hacia el mecanismo de producción de la enfermedad ${ }^{14}$.

Generalmente el compromiso retinal es bilateral y simétrico, sin embargo, en nuestra casuística en 3 pacientes tratados con bevacizumab se observó compromiso retinal desigual (tabla 2), situación que ha sido observada también en otros estudios de retinopatía ${ }^{4,17}$.

Los reportes iniciales de neonatos tratados con bevacizumab presentaron la experiencia del tratamiento en uno o dos ojos ${ }^{13,14,16,18}$, utilizándose en uno de estos reportes la asociación con FCL con respuesta favorable ${ }^{13}$. Sin embargo, en otro estudio un paciente que se trató en etapa 4A plus tuvo una mala respuesta al tratamiento con bevacizumab ${ }^{16}$.

En un ensayo clínico con reducido número de pacientes se utilizó bevacizumab poste- rior al tratamiento convencional con láser, al existir complicaciones locales como hifema o recurrencia de la enfermedad, y luego bevacizumab como monoterapia en casos seleccionados. Estos autores publican la experiencia en 10 niños con 12 ojos tratados, con buena respuesta oftalmológica y adecuada tolerancia clínica, requiriendo reintervención en sólo dos ojos $^{19}$. Este reporte es similar a la experiencia local, en que también se ha utilizado bevacizumab inicialmente como coadyuvante y posteriormente como monoterapia en pacientes seleccionados.

En nuestra experiencia, al usar inicialmente bevacizumab como coadyuvante a la terapia estándar con láser su eficacia fue $100 \%$ al no requerir reintervención, lográndose el propósito por el cual se inició este tratamiento. Posteriormente al utilizar el bevacizumab como monoterapia en casos seleccionados, su eficacia fue de $63,6 \%$, pudiendo tratarse aquellos pacientes que dadas sus condiciones iniciales no era posible la FCL.

En una serie extranjera, en la que se utilizó bevacizumab más láser en forma simultánea como tratamiento de la RDP en etapa umbral, se observó una efectividad comparable a la nuestra, alcanzando un $90,2 \%{ }^{20}$. Un estudio que comparó la administración de bevacizumab antes o después de la FCL, no mostró diferencias de efectividad en el seguimiento de los pacientes a un año plazo ${ }^{21}$.

Recientemente, en el año 2011, se publicó el primer estudio prospectivo multicéntrico randomizado controlado, con un universo de 150 pacientes, comparando la eficacia del bevacizumab versus la fotocoagulación con láser, en la RDP etapa 3+. Este ensayo clínico mostró como resultado un $4 \%$ de recurrencia con bevacizumab como monoterapia y un $22 \%$ en el grupo tratado con FCL. Se concluyó que el bevacizumab intravítreo comparado con la FCL mostró beneficios significativos en la detención de la enfermedad en zona I, pero no en zona II posterior ${ }^{7}$. En nuestro estudio, los 3 pacientes con RDP localizada en zona I tuvieron buena respuesta y no requirieron reintervención.

En el presente ensayo clínico, el bevacizumab se administró en forma intravítrea a una 
dosis única de $0,625 \mathrm{mg}$, cantidad equivalente a la mitad de la dosis utilizada en el ojo de un adulto. En diversos estudios, las dosis utilizadas varían entre $0,4 \mathrm{mg}$ a $1,25 \mathrm{mg}^{9}$. En un estudio experimental, se administró la misma dosis que en nuestra casuística, considerando que el ojo del RN pretérmino es $50 \%$ más pequeño que el ojo adulto, lográndose resultados alentadores, salvo en dos ojos reintervenidos ${ }^{19}$. La presentación original del fármaco que se utilizó en nuestro centro contiene $100 \mathrm{mg}$ de bevacizumab, por lo que utilizándose la dosis mencionada anteriormente, podría obtenerse en condiciones ideales hasta 160 inyecciones de este fármaco al ser adecuadamente fraccionado; resultando en un tratamiento de bajo costo.

La respuesta al fármaco en nuestros pacientes fue satisfactoria sin presentar complicaciones oculares a corto plazo. En la literatura, tanto en las series de casos como en diversas revisiones, se reportan escasas complicaciones, correspondiendo algunas de ellas a hifema, endoftalmitis, desprendimiento de retina o ca$\operatorname{tarata}^{9,22,23}$. Por otra parte, la insuficiente información sobre la seguridad del bevacizumab, tanto local como sistémica, y también a largo plazo ha significado que algunos especialistas se manifiesten cautos con su uso ${ }^{24,25}$. El mismo grupo de investigadores de BEAT-ROP aclara que su universo no fue suficiente como para determinar seguridad en su uso ${ }^{7}$.

Dado el rol angiogénico del VEFG necesario para la formación de nuevas estructuras, entre las que se encuentra el sistema nervioso central y el desarrollo alveolar pulmonar, se podría especular que el bloqueo de esta molécula podría interferir en múltiples procesos, por lo que se hace indispensable el seguimiento rutinario de estos pacientes ${ }^{26,27}$.

Aunque la migración del medicamento hacia otras partes del organismo y sus posibles consecuencias son desconocidas, el bevacizumab es en comparación con otros anticuerpos monoclonales, una molécula grande- alrededor de 150 kD- lo que limitaría su difusión fuera del globo ocular, disminuyendo por tanto la probabilidad de efectos sistémicos ${ }^{28}$. En una revisión de la farmacocinética intravítrea se encontró que la concentración sérica de be- vacizumab fue un $0,8 \%$ de la concentración intraocular ${ }^{29}$, alcanzando este nivel luego de 8 días. Este nivel aumentó al ser el fármaco administrado en ambos ojos, especialmente si se inyecta en ojos de menor edad gestacional ${ }^{30}$. Se ha reportado que una edad gestacional corregida segura para la administración de este fármaco sería a las $34 \pm 6 \mathrm{sem}$. Su uso previo a las 32 sem podría llevar a una inhibición de la angiogénesis de la retina periférica, con pérdida de campo visual ${ }^{31}$. En nuestro estudio, el promedio \pm DS de la edad gestacional corregida al momento de la inyección fue de $34,7 \pm 2$ sem, similar al rango de seguridad reportado ${ }^{31}$.

La vida media descrita para bevacizumab en el vítreo es de $4 \mathrm{sem}$, por lo cual no sería necesaria una nueva intervención antes de este tiempo ${ }^{32}$. Esto no concuerda completamente con nuestra revisión, en la cual 3 ojos debieron ser reintervenidos a las tres semanas del tratamiento inicial con láser por progresión de la RDP (pacientes 8, 14, 16; tabla 1).

Aunque aún no se dispone de suficiente información como para recomendar ampliamente su uso, el tratamiento de la RDP en etapa umbral con bevacizumab intravítreo muestra ventajas respecto al tratamiento convencional con fotocoagulación con láser ${ }^{8}$. Destaca por ser una terapia menos destructiva; más específica según la patogénesis de la enfermedad; con posible menor pérdida de campo visual; y que se puede llevar a cabo sin anestesia general o necesidad de traslado del paciente ${ }^{33}$.

\section{Conclusiones}

El presente ensayo clínico indica que el bevacizumab sería una terapia efectiva en el tratamiento de la RDP umbral en casos seleccionados, presentando como ventaja respecto a la FCL el ser un procedimiento de menor duración, que puede ser realizado en la Unidad de Neonatología, sin necesidad de anestesia general y que respeta más la integridad de la retina. En nuestro centro, considerando un universo de 17 pacientes, se observó una respuesta satisfactoria a la monoterapia con bevacizumab, requiriendo menos de la mitad de los pacientes reintervención con FCL, sin presentar ninguno de ellos complicaciones oculares inmediatas. 
Se hace necesario un seguimiento a futuro de estos pacientes para evaluar efectos locales y sistémicos a mediano y largo plazo.

\section{Referencias}

1.- Hunter DG, Mukai S: Retinopathy of prematurity: pathogenesis, diagnosis and treatment. Int Ophthalmol Clin 1992; 32: 163-84.

2.- Gilbert $C$ : Retinopathy of prematurity: a global perspective of the epidemics, population of babies at risk and implications for control. Early Hum Dev 2008; 84: 77-82.

3.- Gilbert C, Cánovas Emhart R, Kocksch Werner R, Foster FCA: Ceguera infantil en Chile: estudio de escuelas de ciegos/Child blindness in Chile: study of blind schools. Arch Chil Oftalmol 1993; 50: 49-53.

4.- Bancalari A, González R, Vásquez C, Pradenas I: Retinopatía del prematuro: incidencia y factores asociados. Rev Chil Pediatr 2000; 71: 114-21.

5.- Flyn JT, O'Grady GE, Herrera J, Kushner BJ, Cantolino S, Milam $W$ : Retrolental fibroplasias: clinical observations. Arch Ophthalmol 1977; 95: 217-23.

6.- Chen J, Smith LE: Retinopathy of prematurity. Angiogenesis 2007; 10: 133-40.

7.- Mintz-Hittner HA, Kennedy KA, Chuang AZ: Efficacy of intravitreal bevacizumab for stage 3+ retinopathy of prematurity. N Engl J Med 2011; 364: 603-15.

8.- Law JC, Recchia FM, Morrison D, Estes R: Intravitreal bevacizumab (Avastin) as adjunctive treatment for retinopathy of prematurity (ROP). J AAPOS 2010; 14: 6-10.

9.- Micieli JA, Surkont M, Smith AF: A systematic analysis of the off-label use of bevacizumab for severe retinopathy of prematurity. Am J Ophthalmol 2009; 148: 53643.

10.- Shih T, Lindley C: Bevacizumab: an angiogenesis inhibitor for the treatment of solid malignancies. Clinical Therapeutics 2006; 28: 1779-802.

11.- FDA: Avastin Approval History. U.S. Food and Drug Administration. Available at http://www.accessdata. fda.gov/drugsatfda_docs/label/2009/125085s0169lbl. pdf Accessed 07/10/12.

12.- Avery RL, Pearlman J, Pieramici DJ, et al: Intravitreal bevacizumab (Avastin) in the treatment of proliferative diabetic retinopathy. Ophthalmology 2006; 113: 1695. e1-15.

13.- Chung EJ, Kim JH, Ahn HS, et al: Combination of laser photocoagulation and intravitreal bevacizumab (Avastin) for aggressive zone I retinopathy of prematurity. Graefes Arch Clin Exp Ophthalmol 2007; 245: 1727-30.
14.- Travassos A, Teixeira $S$, Ferreira $P$, et al: Intravitreal bevacizumab in aggressive posterior retinopathy of prematurity. Ophthalmic Surg Lasers Imaging 2007; 38: 233-7.

15.- International Committee for the Classification of Retinopathy of Prematurity: The International Classification of Retinopathy of Prematurity revisited. Arch Ophthalmol 2006; 124: 1669-70.

16.- Honda S, Hirabayashi H, Tsukahara Y, et al: Acute contraction of the proliferative membrane after an intravitreal injection of bevacizumab for advanced retinopathy of prematurity. Graefes Arch Clin Exp Ophthalmol 2008; 246: 1061-3.

17.- Prendiville A, Schulenburg WE: Clinical factors associated with retinopathy of prematurity. Arch Dis Child 1988; 63: 522-7.

18.- Kong L, Mintz-Hittner HA, Penland RL, et al: Intravitreous bevacizumab as anti-vascular endothelial growth factor therapy for retinopathy of prematurity: a morphologic study. Arch Ophthalmol 2008; 126: 1161-3.

19.- Roohipoor R, Ghasemi H, Ghassemi F, Karkhaneh R, Riazi-Esfahani M, Nili-Ahmadabadi M: Intravitreal bevacizumab in retinopathy of prematurity: an interventional case series. Graefes Arch Clin Exp Ophthalmol 2011; 249: 1295-301.

20.- Autrata R, Senková K, Holousová M, Krejcírová I, Dolezel Z, Borek I: Effects of intravitreal pegaptanib or bevacizumab and laser in treatment of threshold retinopathy of prematurity in zone I and posterior zone II-four years results. Cesk Slov Oftalmol 2012; 68: 29-36.

21.- Choovuthayakorn J, Ubonrat K: Intravitreal bevacizumab injection in advanced retinopathy of prematurity. $\mathrm{J}$ Med Assoc Thai 2012; 95 S4: 70-5.

22.- Bakri S, Larson T, Edwards A: Intraocular inflammation following intravitreal injection of bevacizumab. Graefes Arch Clin Exp Ophtalmol 2008; 246: 779-81.

23.- Jamrozy-Witkowska A, Kowalska K, Jankowska-Lech I, Terelak-Borys B, Nowosielska A, Grabska-Liberek I: Complications of intravitreal injections-own experience. Klin Oczna 2011; 113: 127-31.

24.- Gole G, Camuglia J, Ells A: Bevacizumab for Retinopathy of Prematurity. N Engl J Med 2011; 364: 2359-62.

25.- Michaud AP, Bauman NM, Burke DK, Manaligod JM, Smith RJH: Spastic diplegia and other motor disturbances in infants receiving interferon-alpha. Laryngoscope 2004; 114: 1231-6.

26.- Thébaud B, Abman S: Bronchopulmonary Dysplasia Where Have All the Vessels Gone? Roles of Angiogenic Growth Factors in Chronic Lung Disease. Am J Respir Crit Care Med 2007; 175: 978-85. 
27.- Katz X: Prematuridad y visión. Rev Med Clin Condes. 2010; 21 (6): 978-83.

28.- Mintz-Hittner HA: Avastin as monotherapy for retinopathy of prematurity. J AAPOS 2010; 14: 2-3.

29.- Bakri SJ, Snyder MR, Reid JM, Pulido JS, Singh RJ: Pharmacokinetics of intravitreal bevacizumab (Avastin). Ophthalmology 2007; 114: 855-9.

30.- Wu WC, Lai CC, Chen KJ, et al: Long-term tolerability and serum concentration of bevacizumab (avastin) when injected in newborn rabbit eyes. Invest Ophthalmol Vis Sci 2010; 51: 3701-8.

31.- Lee JY, Chae JB, Yang SJ, Yoon YH, Kim JG: Effects of intravitreal bevacizumab and laser in retinopathy of prematurity therapy on the development of peripheral retinal vessels. Graefes Arch Clin Exp Ophthalmol 2010; 248: 1257-62.

32.- Beer PM, Wong SJ, Hammad AM, Falk NS, O'Malley $M R$, Khan $S$ : Vitreous levels of unbound bevacizumab and unbound vascular endothelial growth factor in two patients. Retina 2006; 26: 871-6.

33.- Castellanos MA, Schwartz S, Leal R, Chan RV, QuirozMercado $H$ : Pain assessment in premature infants treated with intravitreal antiangiogenic therapy for retinopathy of prematurity under topical anesthesia. Graefes Arch Clin Exp Ophthalmol. 2012 May [Epub ahead of print]. 